\title{
Crafting cover letters
}

\author{
Cover letters are a ubiquitous but hidden part of the publication process. We share our thoughts on the effective \\ and efficient crafting of these letters and their role in our editorial decision-making.
}

C over letters highlighting a study's conclusions and sharing details relevant to the review process are important parts of manuscript submissions, even if they are only ever read by authors and editors. The writing of these letters can, however, seem like an additional burden. In the interest of helping our authors write these letters, we've put together thoughts from the editors at Nature Geoscience.

First off, our decisions to proceed to review are based only on an assessment of the broader relevance, novelty, and importance of a study's main conclusions, following a careful reading of a manuscript and relevant background literature. Manuscripts need to stand on their own - cover letters can provide editors with valuable context, but our decisions are not based on their content. They can, for example, clarify whether a study matches the journal's scope, especially if this is not readily apparent after a fair reading of the manuscript. Outlining the place of conclusions relative to open, important scientific questions or debates within research communities is also helpful.

Don't fret over how to address your letter - a simple 'Dear Editor' is all that's required. They can be addressed to specific editors, especially if you have had a prior interaction. We always aim to match submissions to editors based on subject expertise, but this is not always guaranteed (though editors frequently consult with each other). While it ultimately won't affect our editorial decisions, try to ensure that the letter is addressed to the correct journal before you submit. In some instances, this may not be possible - like when a manuscript is transferred from another Nature Portfolio journal. We of course recognize that manuscripts may have been previously submitted to other journals, but do keep in mind that studies must only be submitted to one journal at a time and our scope may be different.

Keep it concise. This is your chance to have a casual conversation about your work with the editor and a single page is typically enough. Don't worry too much about grammar or tone, we appreciate that many of our authors are not writing in their first language and, as with our reading of manuscripts, we always focus on the scientific content and not the writing style. It's fine to be excited (we appreciate you're talking about the end result of a lot of hard work), but keep hyperbole to a minimum. It is a lot more useful to explain why a study represents an important scientific finding instead of repeatedly declaring it so - again, the novelty and advance of your study should stand on its own. If the work has broader societal or policy relevance beyond the geosciences, that can also be discussed. Keep the focus on the study itself and not the resumes of the authors.

Cover letters are especially important for initial manuscript submissions, but we also strongly encourage their inclusion with resubmissions. They do not have to be long but should bring to the attention of the editor any confidential issues that aren't suitable for the response to reviewers' comments.

References to other studies are not required, but can be useful in some circumstances. Cover letters are the right place to explain how the current study relates to other published or (especially) yet-to-be published work by you or your co-authors, especially in light of our dual publication policies. Please be up-front if the publication of your study will be contingent on publication of another. If this is the case, it is still fine to submit the manuscript but we might ask that the associated manuscript be included in the files sent to reviewers. We consider novelty when choosing which papers to send to review, so a brief discussion of other studies with apparently similar conclusions might be appropriate if the overlap is substantial.

Recommend a few relevant reviewers that cover the range of topics and methods in the study. We may or may not use these recommendations if the paper is sent to review, but it can help expedite the review process regardless. These suggested reviewers should ideally not have published previously with the authors of your study, or at least not for some time, and should not be affiliated with the same institutions as any of the authors on the manuscript.

Feel free to exclude reviewers but keep it reasonable. There is no obligation to provide an explanation for these exclusions, especially if only a handful of names are included. However, we do discourage the wholesale exclusion of entire lab groups and their alumni.

If choosing the option of double-blind peer-review, all identifying information about the authors, including the authorship list, acknowledgments, and author contributions statement, should be removed from the main text and pasted in the cover letter instead.

There is no one-size-fits-all approach to writing cover letters, but the most important thing we hope to see as editors is clear enthusiasm and excitement for your work. $\square$

Published online: 3 September 2021 https://doi.org/10.1038/s41561-021-00824-y 